\title{
The autonomy of demented patients: interviews with caregivers
}

\author{
S-L Ekman, A Norberg University of Umeå, Sweden
}

\section{Authors' abstract}

Tape-recorded semi-structured interviews were conducted with 21 nursing aides and enrolled nurses in the geriatric clinic in Umeå, Sweden. The interviews focused on the difference between the care of demented and non-demented patients and ethical conflicts in dementia care. The results indicate that caregivers have problems in providing the demented patients with opportunities to act autonomously in everyday matters on the ward, mainly due to the difficulty of understanding what the patients wish and the fact that their wishes, when understood, often seem irrational. Measures to provide the demented patients with more opportunities to act autonomously in everyday matters are suggested.

Demented patients gradually lose their ability to make their own decisions as their cognitive impairment increases. This leads to ethical problems in care. Several articles have discussed how to make decisions about such life and death matters as, for instance, when to stop feeding severely demented patients $(1,2)$. There have been discussions whether physicians should act paternalistically in the patient's best interest or whether they should be guided by respect for the patient's past autonomy $(3,4,5,6)$.

Although modern nursing philosophy emphasises the importance of patient participation and shared goals, Wilson-Barnett (7) states that it quite often happens that caregivers in the care of the elderly act for the benefit of the patients without their consent or even against it. This statement is in accordance with our personal experience. It seems important to understand circumstances leading to neglect of patient autonomy in dementia care.

An unpublished observation study of time consumption in a geriatric clinic revealed that most caregivers spent less time with demented than with non-demented patients with the same Activity of Daily Living (ADL) state (8). It seems reasonable to suspect that the reason for this time difference was that caregivers did not spend sufficient time trying to communicate with demented patients to enable them

\section{Key words}

Autonomy; dementia; communication; nursing; ethical conflicts. to learn how to respect their autonomy.

The aim of this study was to investigate whether problems of communication with demented patients contributed to neglect of patient autonomy.

\section{Methods}

The study was carried out at three wards in the geriatric clinic in Umeå, Northern Sweden, the clinic where the above study concerning time consumption was performed. All the twenty-four nursing aides and enrolled nurses who had worked for one to five $(\mathrm{me}=2)$ years at the clinic, and who worked daytime during the three months that the interviewing lasted were interviewed. They were between 21 and $49(\mathrm{me}=25.5)$ years old. There were four males in the group. The nurses' aides and enrolled nurses were assigned patients in a special patient room, while the registered nurses worked according to a task-assignment care system. There were 0.90 caregivers for each of the 24 beds in the clinic. No extra personnel were assigned to the ward during sick leaves or vacations of up to 30 days.

The interviews were semi-structured and concerned the difference between the care of demented and nondemented patients during morning care, feeding, and social interaction; communication with demented patients, and ethical conflicts in dementia care. In this paper only data concerning ethical conflicts will be reported. In order to make the interviews more concrete each interviewee was asked to choose a patient whom she/he mostly took care of. It was characteristic of the patients chosen that they were totally dependent on care and suffered from behaviour disturbances such as screaming, wandering and apathy.

Autonomy was described to the interviewees as the patient's deciding for himself what to do, and also the caregiver's asking him for permission to do things for and with him.

Paternalism was not defined explicitly. The caregivers were instead asked how they felt when deciding for the patients. They were also asked about their use of force in care.

The interviews, which lasted for about one hour each, were tape-recorded and transcribed. Three interviews were excluded because of technical errors. The remaining twenty-one were analyzed by means of 
content analysis and by counting the types of answers. The result was interpreted as conflicts between patient autonomy and paternalism (9).

\section{Results}

The noted fact that caregivers spent less time caring for demented than caring for non-demented patients with the same ADL status (8) was explained by eleven interviewees as being due to the fact that they did not have to communicate with demented patients.

Seventeen interviewees expressed positive attitudes towards the idea of providing the demented patient with autonomy in care. Four expressed negative attitudes; they did not think that demented patients were aware of their being given an opportunity to be autonomous.

The first group, who were positive about autonomy, described two types of problems. First, there was the problem of finding out the patient's wish. Second, there was the problem of the patient's wish to be irrational.

Interviewees described problems both with regard to the patients' expressing their wishes directly and with regard to their own ability to deduce them on the basis of their knowledge of the values and beliefs that the patients had expressed previously. They also complained that they did not know enough about dementia.

The interviewees described their patients as having a limited supply of words. None could use normal sentences. They often gave inadequate and incomprehensible answers. Interviewees complained that they had very little or no eye contact with their patients. The patients' gestures and facial expressions were scarce, and it was difficult to interpret the few expressions there were.

The interviewees also reported that they very seldom asked the patients' relatives about the patients' previous values and beliefs. Detailed questions about the patient's previous values and beliefs were put concerning two areas, namely music and food. When the interviewer asked what kind of music certain patients had liked earlier, eighteen caregivers said that they knew nothing about that. They seemed astonished at the question. Three caregivers thought that all old people liked the same kind of music. The caregivers knew what food the patients preferred only in cases of allergic reactions; in those cases they made notes of what food should be avoided. They also noted special diets. No notations of personal preferences were made.

Interviewees said that the lack of knowledge about their patients' wishes made them feel forced to make decisions for the patients. They tried to think what was in the patients' best interests. Some stressed that there was a risk of stopping trying to treat the patients as autonomous persons, of stopping trying to understand their wishes. All patients were treated in the same way. Deciding for patients became routine. One caregiver said: 'More and more it becomes a routine to decide (for patients). In the end you forget the patient - it is dangerous - sometimes I wonder what on earth I am doing'. There were also caregivers who thought that there were no problems about deciding for patients in this routine way. Some even emphasised that it was convenient for the personnel. 'Of course, it is convenient, isn't it? You can decide, can't you? You don't need discussions and such things.' Others felt like robots: they did not think about it at all.

Sometimes you could understand the patient's wish, interviewees said, but you felt it was irrational. The patients might hurt themselves or others. Then at worst the caregivers felt they had to force their patients. 'It is like an unavoidable bad thing. You have to try to wash a little, haven't you. Do your best. There is constant resistance all the time. It feels hopeless. Interviewees seemed to avoid the term 'force'. They preferred words like 'persuasion' or 'coaxing'.

Interviewees often described how they had to force (persuade or coax) the patient to wash or eat. When they were asked about their reactions to this, the two situations seemed to give rise to different reactions. The dilemma was felt as more acute in regard to feeding than in regard to washing. Nine caregivers did not experience any conflict in regard to force. Twelve reported dilemmas. Interviewees said that these dilemmas were not discussed openly on the wards.

\section{Interpretations}

The dilemmas could be interpreted as conflict between different ethical principles $(9,10)$. Th caregivers tried to justify their actions to themselves The justification was made by means of giving priority to another ethical principle in conflict with autonomy.

In certain situations there was a conflict between giving priority to the principle of autonomy and to the principle of non-maleficence: If you respected the patient's irrational wish he might hurt himself or others. 'I mean, I cannot let her do what she wants to do. She has to do what I want her to do. Otherwise she will hurt herself.'

In other situations they did not know whether they should give priority to the principle of autonomy or to the principle of justice. 'The caregiver has the overall view, while the patient only seeks his own best, therefore it is right that the caregiver decides,' one interviewee said.

Often it was evident that interviewees tried to justify their forcing the patient by stressing the principle of beneficence. 'I do not think much about it (forcing the patient), because I know it is for her own best,' one interviewee stated.

The interpretations are summarised in Figure 1.

\section{Discussion}

The results indicate that caregivers have problems in providing demented patients with opportunities to act autonomously in everyday matters on the wards firstly because of communication problems that make it hard to get to know the patient's wishes and secondly because of lack of methods to get to know the patient's past values and beliefs. This results in caregivers often 


\section{FIGURE 1}

\begin{tabular}{|l|l|}
\hline COMMUNICATION DEFICIT & COMMUNICATION \\
Patient's wish unknown & Patient's wish known \\
Patient's previous values \\
unknown
\end{tabular}

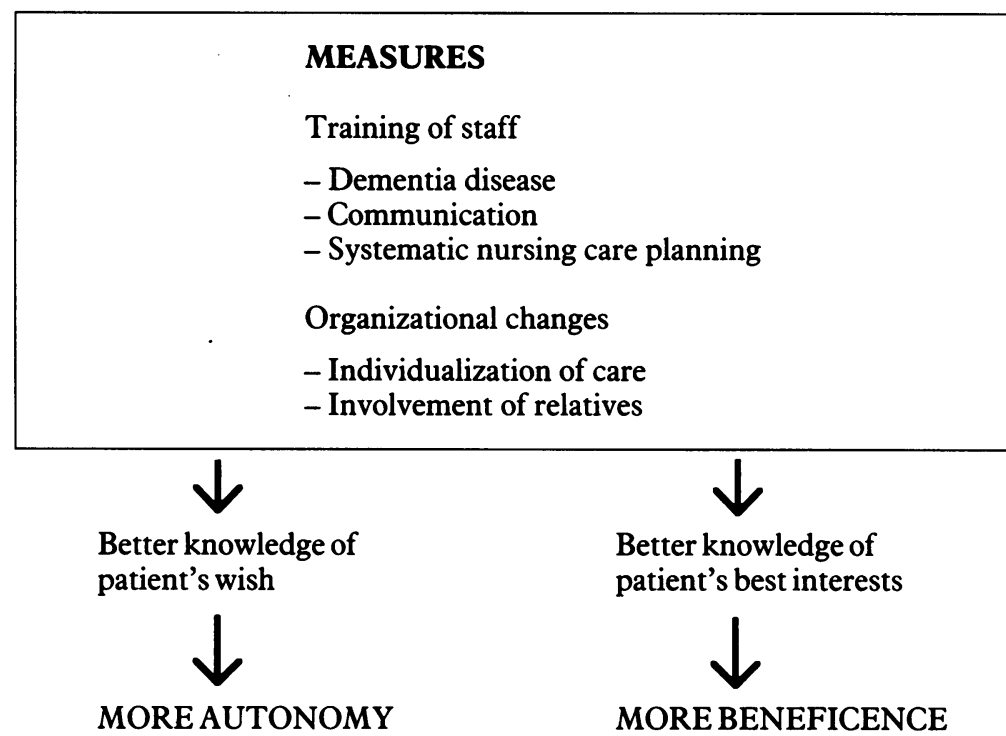

Problems in providing the demented patient with opportunities to act autonomously in everyday matters.

feeling they have to make decisions for their patients. They rationalise this by saying they are doing it for the patients' benefit.

There is a considerable risk that the difficulty involved in finding out the patient's wishes will lead to routine care. This in turn may result in a feeling of indifference among caregivers and consequently harm the patient (11).

Some ethicists, for example Thomasma (12), stress that you should act in the best interests of an incompetent person. Others claim that the incompetent person should have a right to decide for himself, even when his decisions are foolish, as long as they do not cause harm to others (13).

Acting for the patient's best presupposes you know what is best for him. Caregivers' lack of knowledge of the earlier lives of patients interfered with their ability to judge what was best for them. Their lack of knowledge about dementia also made it difficult for them to understand what was best for their patients.

The interviewees reported that they did not want to force their patients although they often felt they had to use mild forms of force in order to act for their patients' best. This is in accordance with a previous finding in nursing homes in Sweden (14). The fact that the caregivers were more prone to accept mild force in 
relation to feeding than in relation to washing could be due to the different consequences of the two acts: if you don't wash yourself nothing disastrous will happen, but if you don't eat you will die.

Force (persuasion and coaxing) always involves danger, even when it might be justified. There is a risk that it might be used in a brutal way. It could mean maleficence - abuse of the patient.

On the basis of our analysis of the interviews we are convinced that autonomy in dementia care could be facilitated by teaching staff about dementia symptoms and training them to communicate with demented patients. This would make them understand their patients' wishes better (15).

Organisational changes should also be made that enable caregivers to understand patients' previous values and beliefs. Each patient should be given individual care: this has been shown to improve caregivers' ability to interpret the communicative cues of severely demented patients (16). Relatives should be involved in care because they know the patient's 'value history'. These changes would improve caregivers' means of interpreting what the patients are trying to communicate and would help them to deduce their wishes from their previous 'value history'. Also, they would make it easier for caregivers to make decisions for their patients' best interests, when no valid information is available on the patients' wishes or when their wishes seem irrational.

\section{Acknowledgements}

This study was supported by grants from Västerbotten County Council and Umeå University. We are grateful to all the medical staff of the geriatric clinic in Umea for their support and to Mrs Åsa Sundh for linguistic revisions.

Sirkka-Liisa Ekman RN, and Astrid Norberg RN PhD are doctoral student and professor respectively in the Department of Advanced Nursing, University of Umea, Umeå, Sweden.

\section{References}

(1) Lynn J, Childress J F. Must patients always be given food and water? Hastings Center Report 1983; 13:17-21.
(2) Olins N J. Feeding decisions for incompetent patients. Fournal of the American Geriatric Society 1986; 34:313317.

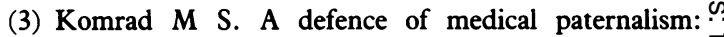
maximising patients' autonomy. Fournal of medical ethics $\overrightarrow{\overline{\vec{D}}}$ $1983 ; 9: 38-44$.

(4) Mariner W K. Decision-making in the care of terminally ill incompetent persons: concerns about the role of the courts. Fournal of the American Geriatric Society 1984; 32:739-746.

(5) McCullough L B. Medical care for elderly patients with diminished competence: an ethical analysis. Fournal of the American Geriatric Society 1984; 32:150-153.

(6) Veatch R M. An ethical framework for terminal care $\overrightarrow{-}$ decisions: a new classification of patients. Fournal of the $\vec{w}$ American Geriatric Society 1984; 32:665-669.

(7) Wilson-Barnett J. Ethical dilemmas in nursing. Fournal of medical ethics 1986; 12:123-126, 135.

(8) Ekman S-L, Eriksson S, Viitanen M. Measuring the need $\vec{\overrightarrow{ }}$ for care - an instrument for the analysis of the work needed in geriatrics (Swe). Unpublished report, 1986.

(9) Beauchamp T L, Childress J F. Principles of biomedical ethics (2nd ed.) New York: Oxford University Press, 1983.

(10) Norberg A, Hirschfeld M. Feeding of severely demented patients in institutions: interviews with caregivers in Israel. Fournal of advanced nursing. 1987; 12:551-557.

(11) Norberg A, Norberg B, Bexell G. Ethical problems in feeding patients with advanced dementia. British medicgl $\overrightarrow{0}$ journal 1980; 281:847-849.

(12) Thomasma D C. Freedom, dependency, and the cares the very old. Fournal of the American Geriatric Socis 1984; 32:906-914.

(13) Shatz D. Autonomy, beneficence, and informed consent: rethinking the connections. Cancerô investigations 1986 ; 4:257-269.

(14) Norberg A, Bäckström $\AA$, Athlin E, Norberg B. Food $\underset{F}{\overrightarrow{7}}$ refusal among nursing home patients as conceptualised by nurses' aides and enrolled nurses. An interview study. Fournal of advanced nursing, in press.

(15) Athlin E, Norberg A. Interaction between the severely demented patient and his caregiver during feeding. A theoretical model. Scandinavian journal of caring science $\overline{\mathbb{D}}$ 1987; 1:117-123.

(16) Athlin E, Norberg A. Caregivers' attitudes to and. interpretations of the behaviour of severely dementedo patients during feeding in a patient assignment care system. International journal of nursing studies 1987;윽 24:145-153. 